diagnostic work-up are essential. We report the case of a 3 year old previously well girl, who was referred to A\&E with shortness of breath. On admission, she was found to be in acute heart failure.

Echocardiography showed a restrictive cardiomyopathy and a very large pericardial effusion which was subsequently drained. A full cardiomyopathy screen was performed but all her metabolic indices were normal. Genetic investigations identified a novel MYH7 variant. The MYH7 gene is usually associated with hypertrophic cardiomyopathy. This patient is the second in the world in whom RCM has resulted from a variant invariant in $M Y H 7$, the first with a phenotypic effect and provides further evidence that this gene is linked with different cardiac phenotypes.

\section{ATYPICAL WHEEZING CASES DURING INFANCY DUE TO GASTRIC VOLVULUS}

doi:10.1136/archdischild-2012-302724.0511

Ö Özdemir, G Direk, A Altunkara. Pediatrics, Istanbul Medeniyet University, Göztepe Research and Training Hospital, Kadiköy, Turkey

Background Gastric volvulus is rarely seen abnormality during childhood. Nonbilious vomiting, abdominal distension and dyspepsia are the most common presenting symptoms. Yet, it may cause reactive airway disease symptoms such as wheezing attacks, because of gastroesophageal reflux, and chronic cough in infants. Surgery after early diagnosis in gastric volvulus resolves symptoms completely and its prognosis is excellent.

Aim Here, two infants having chronic cough and recurrent wheezing attacks, unresponsive to bronchodilators, are presented.

Patients/Methods Physical examination of both cases revealed about 6-month-old male patients having recurrent wheezing, tachypnea, dyspnea and subcostal retractions. Widespread ronchi and sometimes rales were heard on both lungs of the patients. Rest of the examination was normal. In their laboratory evaluations, acute phase reactants, renal and liver function tests were within normal. Immunoglobulin $G$, its subgroups and other immunoglobulins were found to be normal. Sweat tests were normal. High resolution computerized tomography (HRCT) demonstrated minimal mosaic pattern on both lung parenchyma. Bronchoscopy was normal in the first infant. Cranial, abdominal ultrasonography and fundus (eye) examinations showed normal findings in both patients. Cardiac examination and echocardiography were normal. Oesophago-gastro-duodenography showed gastric volvulus, organo-axial and mesentero-axial, in both infants; respectively.

Results After the surgery for gastric volvulus and gastroesophageal reflux in the first case, the symptoms entirely improved. But, the symptoms of second case resolved without surgery.

Conclusion Our cases are being reported to emphasize the necessity of thinking gastric volvulus in the differential diagnosis of atypical wheezing infant, even which it is very nadir cause.

\section{NEONATAL TESTES TORSION: CASE REPORT}

doi:10.1136/archdischild-2012-302724.0512

'M Gündüz, ${ }^{2 M}$ Soran, ${ }^{3 i}$ Çiftçi, 'T Sekmenli, ${ }^{4}$ F Akin. ${ }^{1}$ Pediatric Surgery; ${ }^{2}$ Pediatric Nephrology, Konya Training and Research Hospital; 'Pediatric Surgery, Selcuk University, Selcuklu Medical Faculty; ${ }^{4}$ Pediatric Clinic, Konya Training and Research Hospital, Konya, Turkey

Introduction Torsion of the testes results from twisting of the spermatic cord, which leads to a compromised testicular blood suply and subsequant testicular infarction. The consequant ischemic damage affects long-term testicular morphology and sperm formation.

Case We describe a case of 2 day-old boy who presented with bilateral visible swelling in the scrotum. Physical examination findings showed enlarged, hard, nontender right scrotal mass, and left hydrocele. Doppler ultrasonography demonstrated torsion of right testes and bilateral hydrocele. Exploration was performed using a median raphe incision in the scrotum. Right testes was delivered, detorsed, and placed in warm, moist sponges but it appeared nonviable and necrotic. Right orchiectomy was done. Left testes was normal, it was fixed to the scrotum. Postoperative recovery was uneventful.

Conclusion Perinatal testicular torsion is a rare condition. During newborn examination it must kept in mind if a scrotal mass or swelling occurs.

\section{HEERFORDT'S SYNDROM IN AN ADOLESCENT BOY}

doi:10.1136/archdischild-2012-302724.0513

${ }^{1} \mathrm{~F}$ A Arkan, ${ }^{1} \mathrm{~F}$ Özkan, ${ }^{2} \mathrm{P}$ Işık Ağras, 'T Zengin, 'T Çataklı, 'Y Dallar Bilge. 'Pediatrics; ${ }^{2}$ Pediatric Nephrology, Ministry of Health, Ankara Training and Education Hospital, Ankara, Turkey

Introduction Heerfordt's Syndrome is characterized by bilateral uveitis, facial paralysis, fever and parotitis which is a rarely seen condition in sarcoidosis with neurological manifestations.

Case Report A fifteen year old boy admitted to our pediatric emergency service with fever, vomitting and swellings on his cheeks. Bilateral conjunctivitis, bilateral preauricular swellings, maculopapular rash on his anterior and posterior body areas and uncertain neck stiffness were detected on his physical examination. The rest of the examination was considered as in normal range. His medical history revealed an upper respiratory tract infection one week ago.

Lomber puncture was performed because of patient's neck stiffness. No cell and culture growth were detected on puncture sample. During his observation, left peripheral facial paralysis was developed and bilateral uveitis was detected while his high fever was still persisting.

Whole body gallium 67 scan was performed. Focal accumulations of gallium 67 in both lacrimal glands, parotid and submandibular glands(panda sign) were seen. Because of existence of uveitis, parotitis, left peripheral facial paralysis and fever, the patient was diagnosed as Heerfordt's Syndrome. Steroid (Prednisolone) treatment was started. The regressions of facial paralysis and parotid gland size were noticed after two weeks of the steroid therapy.

Result This case report was chosen to take attention to a rare cause of parotitis

\section{THE AUTOIMMUNE LYMPHOPROLIFERATIVE SYNDROME: A CASE REPORT}

doi:10.1136/archdischild-2012-302724.0514

'L Sfaihi, 'S Kmiha, 'I Maaloul, ${ }^{2}$ B Ben Mustapha, ${ }^{2} \mathrm{~B}$ Barbouch, 'M Hachicha. ' $\mathrm{CHU}$ Hedi Chaker, Sfax; ${ }^{2}$ Institut Pasteur, Tunis, Tunisia

Background The Autoimmune Lymphoproliferative Syndrome (ALPS) is an impairment of lymphocyte apoptosis expressed by generalized non-malignant lymphoproliferation, lymphadenopathy and/or splenomegaly. Majority of patients with ALPS harbor heterozygous germline mutations in the gene for the TNF receptorfamily member Fas (CD 95, Apo-1) which are inherited in an autosomal dominant fashion. Somatic Fas mutations are the second most common genetic etiology of ALPS

Case report We describe a two year old boy who was admitted with hepatosplenomegaly, generalized lymphadenopathy and anemia. Histopathological and immunohistochemical analysis of lymph nodes suggested a lymphoproliferative disorder in large granular lymphocytes. The lymphocyte phenotyping performed in the patient showed an increased population of $\mathrm{T}$ cells $\alpha \beta$ double 